Portland State University

PDXScholar

$1-1-1997$

\title{
Optical imaging of carrier dynamics in silicon with subwavelength resolution
}

\author{
Andres H. La Rosa \\ Portland State University \\ B. I. Yakobson \\ H. D. Hallen
}

Follow this and additional works at: https://pdxscholar.library.pdx.edu/phy_fac

Part of the Physics Commons

Let us know how access to this document benefits you.

\section{Citation Details}

La Rosa, A. H., Yakobson, B. I., \& Hallen, H. D. (1997). Optical imaging of carrier dynamics in silicon with subwavelength resolution. Applied Physics Letters, 70(13), 1656.

This Article is brought to you for free and open access. It has been accepted for inclusion in Physics Faculty Publications and Presentations by an authorized administrator of PDXScholar. Please contact us if we can make this document more accessible: pdxscholar@pdx.edu. 


\title{
Optical imaging of carrier dynamics in silicon with subwavelength resolution
}

\author{
A. H. La Rosa, B. I. Yakobson, and H. D. Hallen ${ }^{\text {a) }}$ \\ Department of Physics, North Carolina State University, Raleigh, North Carolina 27695
}

(Received 18 September 1996; accepted for publication 28 January 1997)

\begin{abstract}
Characteristic rate variations of carrier processes are imaged using near-field scanning optical microscopy. We couple both a visible pump and an infrared probe light through a subwavelength aperture to investigate the interband recombination and intraband diffusion of excess carriers in oxidized silicon. Typical values of the locally measured life time constants agree well with those obtained by conventional space-averaged techniques. Moreover, the images locate defects, reveal variations, and can map the regions in which a recombination process is active. (C) 1997 American Institute of Physics. [S0003-6951(97)02613-2]
\end{abstract}

Carrier relaxation in photoexcited semiconductors proceeds through several, generally overlapping in time, stages: very fast dephasing of the excited states (fractions of picosecond), electron-hole plasma thermalization (1-100 ps), and recombination (fractions of a microsecond for direct and up to milliseconds for indirect semiconductor, like Si). Their active "lifetime" $\tau$ determines the material electrical properties and depends significantly on material quality. A variety of defects are capable of promoting the relaxation and thus reducing the lifetime. A high value of $\tau$ usually indicates lower concentration of these impurities or defects, i.e., recombination centers for carriers. Measurements of $\tau$ and, more generally, of carrier dynamics provide information about the defect distribution, a critical factor for their use in technological applications. The time domain addressed in our study lies therefore in the $\mu$ s to ms range. Furthermore, the continuing trend towards miniaturization of devices brings new challenges for semiconductor studies: the demand for local rather than average material characterization. In this letter we report on high resolution (subwavelength) optical imaging of carrier dynamics in silicon. That is, the carriers are detected locally, and an image is created with the contrast selective to their temporal behavior. Typical values of the locally measured time constants agree well with those obtained by conventional space-averaged techniques. Moreover, the images locate defects, reveal variations, and can map the regions in which a recombination process is active.

In what resembles a modern version of the classic Haynes-Shockley experiment, ${ }^{1}$ the approach we use brings three techniques together to achieve such dynamic imaging: (i) a noncontact and noninvasive measurement of $\tau$ can be obtained with standard "pump probe" techniques involving conventional optics ${ }^{2-4}$ photoexcitation by visible light generates carriers, which scatter infrared (IR) radiation, thereby reducing the IR intensity at the detector. However, when very fine lateral resolution is required, far-field optics encounters a limitation: features smaller than $\sim \lambda / 2$ cannot be resolved. ${ }^{5}$ The wavelength $\lambda$ of the radiation being used in these optical lifetime studies is typically $1-10 \mu \mathrm{m}(1.15 \mu \mathrm{m}$ here) infrared light probing carriers excited by green or red light. (ii) High spatial resolution can be attained with nearfield scanning optical microscopy (NSOM), ${ }^{6,7}$ in which the

a)Electronic mail: Hans-Hallen@ncsu.edu light is geometrically confined by a small aperture placed in close proximity to the sample. Boundary conditions on Maxwell's equations make the resolution in the near field ${ }^{8}$ a function of the physical size of the aperture $(\sim 100 \mathrm{~nm}$ here $)$ and its distance to the sample surface $(\sim 10 \mathrm{~nm})$, not of the wavelength of the light employed. In order to ensure high lateral resolution and to avoid a mismatch in the illuminated sample regions, both the visible and IR light are coupled through the same aperture." ${ }^{9,10}$ (iii) The addition of an "amplitude modulation" makes this detection sensitive to the relaxation time of carriers: at low frequencies a large signal is observed, but at high modulation frequencies the carrier relaxation cannot keep pace, so the carrier concentration remains almost constant resulting in almost no change in IR transparency. Timeresolved optical semiconductor characterization techniques with fine spatial resolution offer unique opportunities for studies of the dynamics of carriers and the nondestructive evaluation (NDE) of semiconductors. The purpose of this letter is to demonstrate measurements of the lifetime of excess carriers in semiconductors with high spatial resolution in a noncontact optical configuration. The technique can be used to locate any defects that reduce that lifetime locally. It results in a map of carrier dynamics that identifies the dominant processes as a function of position on the sample.

A schematic of the microscope and detection geometry ${ }^{9}$ (with the tip greatly enlarged) is shown on the left side of Fig. 1. The aperture is fabricated by coating the sides of a sharpened optical fiber with aluminum. ${ }^{11,12}$ A scanning electron microscope is used to characterize tip shapes and aperture sizes. An image is produced by rastering the tip over an array of points $(100 \times 100$ here $)$, collecting data at each point (45 ms averaging per point here), and converting the data to an image in a computer. In addition to the image constructed from the optical data, a topographic image of the sample surface is generated. The latter is acquired by recording the output of a lateral force tip-sample feedback system, which ensures that the tip remains a fixed distance (typically 10 $\mathrm{nm}$ ) from the sample during scanning. We record the change in the transmitted IR signal as the visible light is switched on and off using a lock-in amplifier, as shown on the right side of Fig. 1. The change in the IR signal at the frequency of visible light switching is related to the carrier response time. We note that the variation of the visible power results in some thermal variation of the probe which can also induce a 


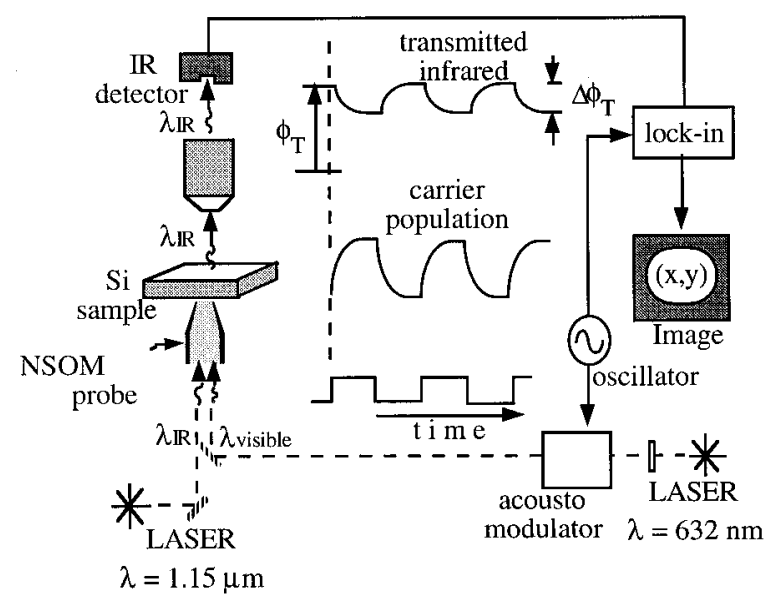

FIG. 1. Schematic of the experiment that extracts information about the local dynamics of excess carriers in a semiconductor. Left side: Optical path of the IR radiation through the microscope. Center: A timing diagram of the process. The visible light creates carriers which decay over their lifetime. An increase in carrier population causes a decrease in the IR transmission. Right: Diagram of the synchronous detection of the transmitted IR signal. An image is constructed as the probe is scanned across the sample.

change in the IR. Our detailed studies ${ }^{13}$ show that this time scale is relatively large, close to tens of ms. The difference in time scales allows separation of the probe effect from the kinetic processes of carrier relaxation in silicon. ${ }^{14}$

An example of imaging with this technique on an oxidized silicon sample ${ }^{15}$ is shown in Fig. 2. On the left is the topography showing a terraced surface, in the center the variation of the nearly constant IR transmission with the visible light off, and on the right the IR variation as the visible light is switched (related to carrier lifetime). Regions with shorter $\tau$ are able to recover more quickly and thus show a larger IR variation. Note in Fig. 2(c) that the carrier lifetime is reduced near some of the topographic steps seen in Fig. 2(a). This is expected since the carrier lifetime in silicon is dominated by surface defects, of which steps are a clear example.

With the high NSOM resolution one must treat carefully the concept of any local property of excess electrons, such as lifetime. This is due to the high mobility of the electrons and holes. Indeed, a formal definition of the lifetime $\tau(\mathbf{r})$ at certain location $\mathbf{r}$ tells how fast a carrier is expected to recom-

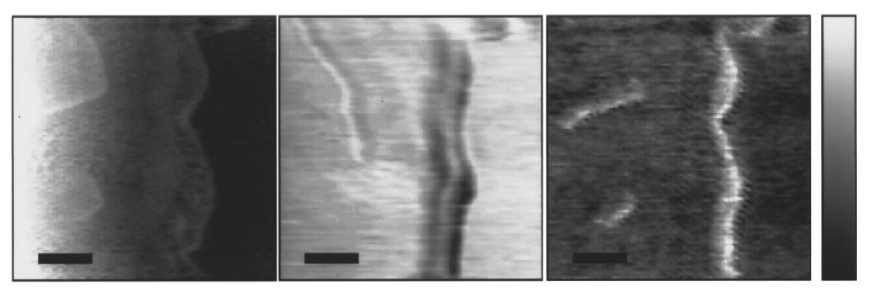

(a)

(b)

(c)

FIG. 2. Three images show the same $20-\mu \mathrm{m}$-sq region of an oxygen terminated silicon surface with different contrast methods. The bars are $4 \mu \mathrm{m}$ long. (a) A topographic image with a $240 \mathrm{~nm}$ vertical range, white higher. (b) The infrared transmission $(0.71 \mathrm{nW}$ range with a $\sim 80.0 \mathrm{nW}$ dc background subtracted) shows regions of contrast, white more intense. (c) The time-resolved image of the change in IR amplitude as the visible light is switched reveals regions near multiple step defects which have a faster recombination rate (white). The range is $0.063 \mathrm{nW}$. bine provided it stays at the same place. In reality though, the fidgety carriers dwell around a substantial spatial domain before the recombination occurs. With a diffusion constant $D=10 \mathrm{~cm}^{2} / \mathrm{s}$ and $\tau>1 \mu \mathrm{s}$, they travel as much as $\sqrt{D \tau}$ $>30 \mu \mathrm{m}$. Therefore, the lifetime may seem to be a quantity intrinsically "averaged" over a relatively large area of a sample. Is it possible then to talk about a local lifetime, and to learn about the defects with a resolution on a nanometer scale? The answer is positive, as the data above demonstrate, and as can be understood from a simple analysis of the local concentration of carriers.

The qualitative picture is that the excess carriers which are locally created by the visible light rapidly diffuse into a region larger than the size of an image and form a distribution $n$, weakly peaked under the probe. This distribution reflects the local lifetime through its variations $\Delta n$, which are sensed at high spatial resolution by the IR. Although one might first guess that $\Delta n$ would vary on a length scale given by the diffusion length, it is not the case. Rather, $\Delta n$, and hence the resolution, varies on a length scale given by $R$, the size of the "object" or inhomogeneity in the sample. As an example, let us consider some domain of radius $R$ within which the carriers recombine by $\delta(1 / \tau)$ faster than elsewhere. A faster decay will lead to a variation in the local carrier density $\Delta n$. This gradient causes an influx of carriers $\sim D \cdot R \cdot \Delta n$ from the surrounding volume. In steady state, the decay balances the influx, and the concentration deviates from a uniform by $\Delta n / n_{\infty}=-\delta(1 / \tau)\left(R^{3} / 3 D\right) r^{-1}$ at a distance $r>R$ from the center; the function is localized for small $R$ (small defect areas) which are of interest here. In the defect-rich area $\Delta n / n_{\infty} \sim R^{2} \cdot \delta\left(\tau^{-1}\right) / 3 D$, which produces a detectable change in IR absorption. ${ }^{16}$ The change in the IR signal is inversely proportional to the mobility and decreases for smaller feature sizes, thus passing the problem of high resolution to the one of signal level because small $\Delta n$ 's have to be distinguished.

A variation of the frequency of the visible light switching at a particular point on the sample adds another dimension, allowing the quantitative determination of the carrier lifetime. If the visible light is switched very rapidly, the carrier concentration does not have time to react and so the IR transmission will not change. At the opposite extreme, at very low visible light switching frequencies, the IR signal will change dramatically. The crossover point will be where the lifetime of the carriers is equal to the period of visible light switching. In Fig. 3(a) we show two plots of the IR signal variation as a function of frequency. The data shown with circles were measured with the probe near the center of the region imaged in the Figs. 3(b) $-(\mathrm{d})$; that shown by triangles was from the sample shown in Fig. 2. These data represent typical measurements from samples with lifetimes, averaged over square $\mathrm{mm}$, measured by a laser/microwave method $^{17}$ to be $1.62 \mathrm{~ms}$ and $13.6 \mu$ s, respectively. The difference in lifetime is presumably due to the more complete bonding, hence there are fewer surface recombination sites with oxygen passivation. The agreement with that obtained from the knee frequency, $f=1 /(2 \pi \tau)$, illustrates the quantitative capabilities of this technique, in addition to its resolution.

A close examination of the data in Fig. 3(a) indicates 
(a)
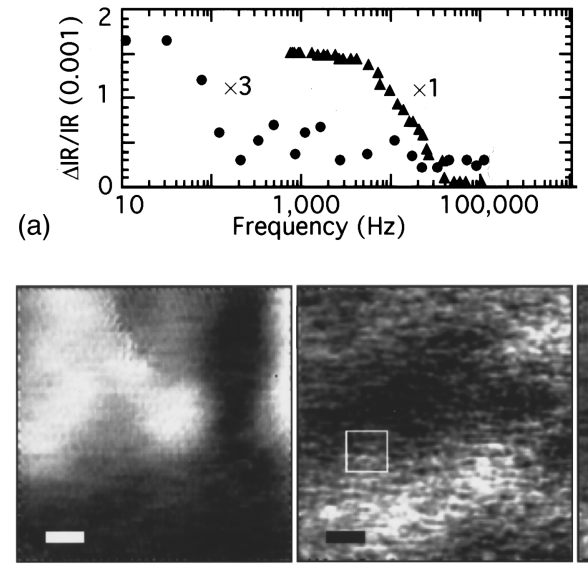

(b)

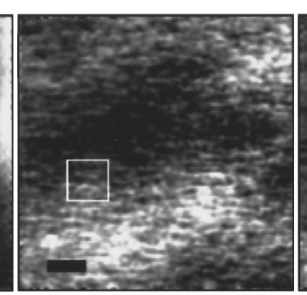

(c)

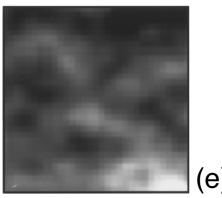

(e)
FIG. 3. (a) Plots of the relative IR signal change vs the visible light switching frequency are shown for two different samples. The samples with data as triangles and circles have effective lifetimes estimated from the knee of 20 $\mu$ s and $1.6 \mathrm{~ms}$, and images shown in Figs. 2 and 3, respectively. (b) $-(\mathrm{d})$ The effects of varying the visible light switching frequency in $7.5-\mu \mathrm{m}$-sq images of nearly the same area on the "circle" sample, with $1 \mu \mathrm{m}$ size bars. (b) An image of the IR signal change as the visible light is switched on and off at $100 \mathrm{~Hz}$ with $0.27 \mathrm{nW}$ range. (c) Same as in (b), but at a frequency of $2 \mathrm{kHz}$, $0.03 \mathrm{nW}$ range. (d) At $20 \mathrm{kHz}$ modulation, $0.03 \mathrm{nW}$ range. (e) Detail from image (d) approximately one wavelength (of $1.15 \mu \mathrm{m} \mathrm{IR}$ ) square, with 0.02 $\mathrm{nW}$ range. The boxes in (c) and (d) show the corresponding area.

that the signal does not vanish at high frequencies, but rather falls to another plateau. This is indicative of a faster serial process preceding the relaxation to the valence band: possibly a diffusion of excess carriers out of the small illuminated spot with characteristic time $a^{2} / D$. The two curves shown for two different samples both have a plateau at low frequencies, a knee near the inverse lifetime, and a low signal at higher frequencies. It is interesting to compare an image taken with a visible switching frequency just below the knee to one taken at a frequency for which the slower process does not respond. This should allow imaging of the two processes independently, and is shown in the remainder of Fig. 3 . The topography in this region is flat, and thus only the lifetime-related images are shown. The slower process, Fig. 3(b), clearly has a different spatial distribution than the faster process, Figs. 3(c) and 3(d). It is interesting to note an approximate reversal of contrast between the images at lower [Fig. 3(b)] and higher [Figs. 3(c) and 3(d)] frequencies. At low frequencies, a larger signal is obtained at regions with long $\tau$ in relatively defect-free areas where the reduced scattering allows faster diffusion. This leads to a shorter time $a^{2} / D$ so that smaller signals are measured at increased modulation frequency, when $1 / \tau<f<D / a^{2}$. It is also interesting to note that the general features of the images at increasingly higher visible light modulation frequencies do not change. This is expected since they all are dominated by the same fast process as is evident in Fig. 3(a). The finer features show some variation with frequency, presumably due to the detection of the residual slower process, but remain identifiable. The resolution of these features is limited by the aperture size, and the smallest feature is clearly finer than the wavelength of light, Fig. 3(e).

In conclusion, we have successfully imaged the spatial distribution associated with processes characterized by different carrier lifetimes. We have shown that lifetime measurements taken in a NSOM on a very local scale compare well with the spatial-averaging techniques widely used. We have shown that local lifetime measurements can be used to provide an image of defect locations near a semiconductor surface. Quantitative mapping of the carrier lifetime at such unprecedented (subwavelength) resolution is now possible. Such measurements will give new insights into carrier transport and recombination processes in all types of semiconductors.

This work was supported by U.S. Army Research Office Grant Nos. DAAH04-95-1-380 and DAAH04-93-G-0194

${ }^{1}$ J. R. Haynes and W. Shockley, Phys. Rev. 81, 835 (1951); S. M. Sze, Physics of Semiconductor Devices (Wiley, New York, 1969).

${ }^{2}$ J. Waldmeyer, J. Appl. Phys. 63, 1977 (1988).

${ }^{3}$ D. L. Polla, IEEE Electron. Device Lett. EDL-4, 185 (1983).

${ }^{4}$ G. Bohnert, R. Hacker, and A. Hangleiter, J. Phys. (France) Colloq. 4, 617 (1988).

${ }^{5}$ J. W. Goodman, Introduction to Fourier Optics (McGraw-Hill, New York, 1968)

${ }^{6}$ D. W. Pohl, W. Denk, and M. Lanz, Appl. Phys. Lett. 44, 651 (1984).

${ }^{7}$ E. Betzig and J. K. Trautman, Science 257, 189 (1992).

${ }^{8}$ E. Betzig, J. K. Trautman, T. D. Harris, J. S. Weiner, and R. L. Kostelak, Science 251, 1468 (1991).

${ }^{9}$ A. H. La Rosa, C. L. Jahncke, and H. D. Hallen, Proc. SPIE 2384, 101 (1995).

${ }^{10}$ A. La Rosa, C. L. Jahncke, and H. D. Hallen, Ultramicroscopy 57, 303 (1995).

${ }^{11}$ E. Betzig, Principles and Applications of Near-Field Scanning Optical Microscopy (NSOM) (Kluwer, Acr-et-Senans, France, 1992), Vol. 1-7, p. 15.

${ }^{12}$ B. I. Yakobson, P. J. Moyer, and M. A. Paesler, J. Appl. Phys. 73, 7984 (1993).

${ }^{13}$ A. H. La Rosa, B. I. Yakobson, and H. D. Hallen, Appl. Phys. Lett. 67, 2597 (1995).

${ }^{14}$ H. D. Hallen, B. I. Yakobson, A. La Rosa, and M. A. Paesler, Proc. SPIE 2535, 34 (1995).

${ }^{15}$ The silicon samples used were phosphorus doped $\left(4 \times 10^{14}\right.$ atoms $\left./ \mathrm{cm}^{3}\right)$, $n$-type Czochralski silicon wafers. The sample surfaces were cleaned with a HF solution prior to silicon dioxide growth. The sample shown in Figs. 2 and 3(a) had a thin, native oxide. The sample shown in Fig. 3 was covered with a 60-nm-thick oxide grown in a commercial furnace with an oxygen ambient.

${ }^{16}$ For example, with $\tau=1 \mu \mathrm{s}, D=1 \mathrm{~cm}^{2} / \mathrm{s}$ and $R=200 \mathrm{~nm}, \Delta n / n_{\infty}=10^{-4} \cdot \tau$ is expected to be even shorter locally, although the average value can be longer.

${ }^{17}$ F. Shimura, T. Okui, and T. Kusama, J. Appl. Phys. 67, 7168 (1990). 